\title{
Exosomes Secreted from CXCR4 Overexpressing Mesenchymal Stem Cells Promote Cardioprotection via Akt Signaling Pathway following Myocardial Infarction
}

\author{
Kai Kang, ${ }^{1}$ Ruilian Ma, ${ }^{1,2}$ Wenfeng Cai, ${ }^{1}$ Wei Huang, ${ }^{1}$ \\ Christian Paul, ${ }^{1}$ Jialiang Liang, ${ }^{1}$ Yuhua Wang, ${ }^{1}$ Tiejun Zhao, ${ }^{3}$ Ha Won Kim, \\ Meifeng Xu, ${ }^{1}$ Ronald W. Millard, ${ }^{4}$ Zhili Wen, ${ }^{5}$ and Yigang Wang ${ }^{1}$ \\ ${ }^{1}$ Department of Pathology and Laboratory Medicine, College of Medicine, University of Cincinnati Medical Center, \\ Cincinnati, OH 45267, USA \\ ${ }^{2}$ Department of Cardiology, The People's Hospital of Sanya, Sanya, Hainan 572000, China \\ ${ }^{3}$ Department of Urinary Surgery, Xining City Hospital, Qinghai 810000, China \\ ${ }^{4}$ Department of Pharmacology and Cell Biophysics, College of Medicine, University of Cincinnati Medical Center, \\ Cincinnati, $\mathrm{OH} 45267$, USA \\ ${ }^{5}$ Infection Hospital of Nanchang University, Nanchang, Jiangxi 330002, China
}

Correspondence should be addressed to Zhili Wen; wenzi@ucmail.uc.edu and Yigang Wang; wanyy@ucmail.uc.edu

Received 4 December 2014; Revised 16 January 2015; Accepted 21 January 2015

Academic Editor: Daniele Avitabile

Copyright (c) 2015 Kai Kang et al. This is an open access article distributed under the Creative Commons Attribution License, which permits unrestricted use, distribution, and reproduction in any medium, provided the original work is properly cited.

Background and Objective. Exosomes secreted from mesenchymal stem cells (MSC) have demonstrated cardioprotective effects. This study examined the role of exosomes derived from MSC overexpressing CXCR4 for recovery of cardiac functions after myocardial infarction (MI). Methods. In vitro, exosomes from MSC transduced with lentiviral CXCR4 (Exo ${ }^{\text {CR4 }}$ ) encoding a silencing sequence or null vector were isolated and characterized by transmission electron microscopy and dynamic light scattering. Gene expression was then analyzed by qPCR and Western blotting. Cytoprotective effects on cardiomyocytes were evaluated and effects of exosomes on angiogenesis analyzed. In vivo, an exosome-pretreated MSC-sheet was implanted into a region of scarred myocardium in a rat MI model. Angiogenesis, infarct size, and cardiac functions were then analyzed. Results. In vitro, Exo ${ }^{\mathrm{CR} 4}$ significantly upregulated IGF-1 $\alpha$ and pAkt levels and downregulated active caspase 3 level in cardiomyocytes. Exo ${ }^{\text {CR4 }}$ also enhanced VEGF expression and vessel formation. However, effects of Exo ${ }^{C R 4}$ were abolished by an Akt inhibitor or CXCR4 knockdown. In vivo, Exo ${ }^{\mathrm{CR} 4}$ treated MSC-sheet implantation promoted cardiac functional restoration by increasing angiogenesis, reducing infarct size, and improving cardiac remodeling. Conclusions. This study reveals a novel role of exosomes derived from MSC ${ }^{\mathrm{CR} 4}$ and highlights a new mechanism of intercellular mediation of stem cells for MI treatment.

\section{Introduction}

Myocardial infarction (MI), resulting from the interruption of blood supply to the heart, is a primary target for experimental stem/progenitor cell-based therapies. Although the therapeutic effect of mesenchymal stem cells (MSC) has been attributed to their differentiation into reparative or replacement cell types [1], the therapeutic importance of cardiovascular lineage remains to be elucidated. CXCR4, a Gprotein-coupled 7-transmembrane receptor, in conjunction with its primary ligand stromal cell-derived factor- (SDF-) $1 \alpha$ serves as a major regulator of stem/progenitor cell activities. The importance of SDF-1 $\alpha /$ CXCR 4 signaling has been documented in CXCR4 knockout rats, which die in utero. This indicates a fundamental developmental role for this receptorligand axis [2]. In previous studies from our laboratory, 
MSC were genetically engineered using ex vivo adenoviral transduction to overexpress CXCR4 in MSC (MSC ${ }^{\mathrm{CR} 4}$ ) [3]. Those studies determined that $\mathrm{MSC}^{\mathrm{CR} 4}$ secreted multiple cytokines such as vascular endothelial growth factor (VEGF) and insulin-like growth factor- (IGF-) $1 \alpha$ in response to hypoxia, augmenting endogenous regenerative processes [3].

Exosomes are naturally occurring membrane-bound nanovesicles $(50-100 \mathrm{~nm})$ which play a role in the selective release of membrane or cytosolic proteins, RNAs, and/or microRNAs (miRNAs), mediating some aspects of cell-to-cell signaling [4]. However, generally speaking these exosomes are not technically "responsible for the selective release" of signaling molecules, as they do not "select" the molecular cargo themselves; they just act as transporters. Recently, A2B1 (hnRNPA2B1) has been proved to artificially load selected small regulatory RNAs into exosomes through recognition of specific short motifs [5]. Exosomes are formed in intracellular vesicular bodies of most cells and released from the cell when multivesicular bodies fuse with the plasma membrane [6]. Previous studies indicated that exosomes released from progenitor cells contain secreting paracrine factors that stimulate neovascularization, thereby mediating cardiac protection during myocardial ischemia/reperfusion injury [7]. The current study is focused on determining the effects of employing a cell-patch containing isolated and purified exosomes from MSC ${ }^{\mathrm{CR} 4}$ in a rat model of myocardial infarction (MI). Patches were implanted into the infarcted zone, and multiple techniques were used to study the engraftment levels and changes in blood vessel formation. This study then used those results to investigate the mechanistic participation of exosomes on the effects of MSC ${ }^{\mathrm{CR} 4}$ after MI and to derive any expression changes in proangiogenic factors including VEGF and IFG- $1 \alpha$. The hypothesis was that exosomes derived from $\mathrm{Exo}^{\mathrm{CR} 4}$ would release their angiogenic factors into the implanted cell sheet, leading to restoration of cardiac functions through angiogenesis, both inside the myocardial infarct region and outside the epicardium (cell sheet).

\section{Material and Methods}

2.1. Isolation and Culture of Rat Bone Marrow MSC and Neonatal Cardiomyocytes (CM). Experiments using animals or animal-derived materials were conducted in accordance with the Guide for the Care and Use of Laboratory Animals (NIH Publication number 85-23, revised in 1996) and under guidelines and protocols approved by the University of Cincinnati Institutional Animal Care and Use Committee. MSC from healthy 8-week-old Sprague-Dawley (SD) rats were isolated from bone marrow aspirates as described previously $[3,8]$. Passage $2-4$ MSC were used in the study. MSC were cultured in high glucose Dulbecco's modified Eagle's medium (DMEM) containing 10\% fetal bovine serum (FBS) and $1 \%$ antibiotics (streptomycin and penicillin) at $37^{\circ} \mathrm{C}$ in humid air with $5 \% \mathrm{CO}_{2}$. When the cell population reached 70-80\% confluence, all cell experiments were performed without serum or antibiotics and repeated at least three times. Neonatal CM was isolated from ventricles of 2-day-old neonatal rats using neonatal CM isolation kit (Worthington
Biochemical) as described [9]. The cells were maintained in Dulbecco's modified Eagle's medium (DMEM medium, Hyclone) with 10\% FBS.

2.2. Transfection of MSC with Lentiviral Constructs. Lentiviral vector backbone pGreenPuro shRNA (pGP) was purchased from System Biosciences (Mountain View, CA). The multiple cloning site of pGP between BamHI and EcoRI restriction enzyme sites was replaced with the siRNA template designed by us to construct the shRNA vector pGPsiR per manufacturer's protocol. The lentiviral vector overexpressing CXCR4 was described in our previous publication [10]. To produce lentivirus for use in MSC transfection, 293NT producer cells (System Biosciences) were transiently transfected with pGP-siR and pGP-null by pPACKH1 HIV Lentivector Packaging Kit (System Biosciences). Viral supernatant was harvested and added to the host cell for 72 hours for viral genome integration into MSC genome. MSC in this study were divided into the following groups: (1) MSC transfected with null lentivirus vector $\left(\mathrm{MSC}^{\mathrm{Ctrl}}\right)$; (2) MSC transfected with lentiviral vector to overexpress CXCR4 $\left(\mathrm{MSC}^{\mathrm{CR} 4}\right)$; (3) MSC transfected with siRNA against CXCR4 $\left(\mathrm{MSC}^{\mathrm{siCR} 4}\right)$ to knock down CXCR4 expression.

2.3. Exosome Isolation from Conditional Medium. All cells were grown in Roswell Park Memorial Institute (RPMI) medium supplemented with 5\% of exosome-depleted FBS (dFBS) (Systems Biosciences) and 1\% penicillin/streptomycin (Invitrogen). MSC were seeded in $100 \mathrm{~mm}$ cell culture dish at $2 \times 10^{5}$ cells/dish. After allowing cells to attach overnight, the medium was replaced and the cells were cultured for 3 days in the fresh medium for approximately $80 \%$ confluence. Exosomes were subsequently isolated from conditioned medium using ExoQuick-TC exosome purify reagent (Systems Biosciences) as manufacturer's protocol. The resulting exosome pellets were resuspended in approximately $200 \mu \mathrm{L}$ PBS and stored at $-80^{\circ} \mathrm{C}$ for subsequent quantification (using BioRad Protein Assay Dye Reagent) and for molecular analysis. Exosomes in this study were divided into the following groups: (1) exosomes isolated from $\mathrm{MSC}^{\mathrm{Ctrl}}\left(\mathrm{Exo}^{\mathrm{Ctrl}}\right)$; (2) exosomes isolated from $\mathrm{MSC}^{\mathrm{CR} 4}\left(\mathrm{Exo}^{\mathrm{CR} 4}\right)$; (3) exosomes isolated from $\mathrm{MSC}^{\mathrm{siCR} 4}\left(\mathrm{Exo}^{\mathrm{siCR} 4}\right)$.

2.4. Transmission Electron Microscopy (TEM) Assay. Exosome pellet was fixed by $2 \%$ glutaraldehyde and $2 \%$ paraformaldehyde (PFA) in 0.1 M Sorensen's phosphate buffer for 3 hours as described [10]. After fixation in 1\% osmium tetroxide $\left(\mathrm{OsO}_{4}\right)$ for $30 \mathrm{~min}$, exosomes were washed and dehydrated in graded series of $50 \%, 70 \%, 80 \%, 90 \%$, and $100 \%$ ethanol and embedded in Epon 812. Ultrathin sections ( $60 \mathrm{~nm}$ ) were cut with a Leica Ultracut UCT ultramicrotome, stained with uranyl acetate and Reynolds lead citrate, and viewed with a transmission electron microscope (JEOL JEM1230).

2.5. Internalization of Exosomes. Exosomes were prelabeled with PHK67 (Sigma) as described [11] and then cocultured 
with CM in DMEM containing 10\% FBS for 48 hours. The uptake of exosomes by CM was stopped by washing in cold PBS, followed by fixation in 4\% PFA for determination of MSC exosome-transduced CM using Olympus BX41 microscope equipped with CCD (MagnaFire, Olympus) camera.

2.6. TUNEL Assay. CM treated with various exosomes were exposed to a hypoxic condition $\left(\mathrm{O}_{2} / \mathrm{CO}_{2}\right.$ incubator-MCO$18 \mathrm{M}$, Sanyo) at $37^{\circ} \mathrm{C}$ with $1 \% \mathrm{O}_{2}: 5 \% \quad \mathrm{CO}_{2}: 94 \% \mathrm{~N}_{2}$ for 24 hours. Then the cells from various treatment groups were fixed with $4 \%$ paraformaldehyde for 30 minutes at room temperature. TUNEL was performed using DeadEnd Fluorometric TUNEL System (Promega) as per instructions of the manufacturer. Nuclei were stained with DAPI for 5 minutes at room temperature. The numbers of TUNEL positive nuclei were determined after counting the cells in randomly selected microscopic fields at 20x using fluorescence microscope (BX41, Olympus). Images were recorded using a digital camera and analyzed using MagnaFire 2.1 software.

2.7. Tube Formation Assay. The assay was performed with a tube formation assay kit (Chemicon) according to the manufacturer's instructions [12]. Briefly, the formation of capillary-like structures was assessed in a 24-well plate using growth factor-reduced matrigel. Human umbilical vein endothelial cells (HUVEC) $\left(3 \times 10^{4}\right.$ cells/well) were seeded on the top of solidified matrigel $(500 \mu \mathrm{L} /$ well $) 2 \mathrm{hr}$. prior to exosomes $(100 \mu \mathrm{L} /$ well $)$ treatment. The HUVEC were treated as following groups: (1) HUVEC + Exo ${ }^{\mathrm{Ctrl}}$, HUVEC treated with Exo ${ }^{\mathrm{Ctrl}}$; (2) HUVEC + Exo ${ }^{\mathrm{CR} 4}$, HUVEC treated with Exo ${ }^{\mathrm{CR} 4}$ (3) HUVEC + Exo ${ }^{\text {siCR4 }}$, HUVEC treated with $\mathrm{Exo}^{\text {siCR4}}$; (4) HUVEC + Exo ${ }^{\mathrm{Ctrl}}+\mathrm{LY}, \mathrm{HUVEC}+\mathrm{Exo}^{\mathrm{Ctrl}}$ group treated with additional PI3K inhibitor LY294002 (10 $\mu \mathrm{M}) ;(5)$ $\mathrm{HUVEC}+\mathrm{Exo}^{\mathrm{CR} 4}+\mathrm{LY}$, HUVEC $+\mathrm{Exo}^{\mathrm{CR} 4}$ group treated with additional LY294002 $(10 \mu \mathrm{M})$. After $16 \mathrm{hr}$, the total tube length was quantified using software Image-Pro Plus 5.1. LY294002 was purchased from Cell Signaling Technology.

2.8. Western Blotting. Briefly, MSC and derived exosomes were lysed in lysis buffer, pH 7.4 (50 mM HEPES, $5 \mathrm{mM}$ EDTA, and $50 \mathrm{mM} \mathrm{NaCl}$ ), $1 \%$ Triton X-100, protease inhibitors $(10 \mu \mathrm{g} / \mathrm{mL}$ aprotinin, $1 \mathrm{mM}$ phenylmethylsulfonyl fluoride, and $10 \mu \mathrm{g} / \mathrm{mL}$ leupeptin), and phosphatase inhibitors (50 mM sodium fluoride, $1 \mathrm{mM}$ sodium orthovanadate, and $10 \mathrm{mM}$ sodium pyrophosphate). The protein samples ( $40 \mu \mathrm{g})$ were electrophoresed using SDS-polyacrylamide gel and electroimmunoblotted as described [8]. After protein transfer, the samples on PVDF membranes were visualized using an enhanced chemiluminescence system (Invitrogen), exposed to X-ray film, and then quantified by laser scanning densitometry. The specific antibodies used for detection of antigens of interest are listed in the following: anti-CXCR4 (Millipore), anti-Akt, anti-pAkt (Cell Signal Technology), anti-IGF-1 $\alpha$, anti-CD9, anti-CD63, and anti- $\beta$-actin antibody (Santa Cruz Biotechnology).
2.9. Quantitative RT-PCR ( $q P C R$ ). The cells were collected for total RNA extraction using TRIzol reagent (Life Technologies). Total RNA was used for reverse transcription in a miScript II RT kit (Qiagen). The primer sequences used were listed as follows: VEGF forward primer $5^{\prime}-\mathrm{GGC}$ AGCTTGAGTTAAACGAAC-3', reverse primer $5^{\prime}$-TGGTTGGTGTGA-CATGGTTAATCGGTC- ${ }^{\prime} ; \beta$-actin forward primer $5^{\prime}$-TGTCATCCTCCCAATCCCTCAGAA-3', reverse primer $5^{\prime}$-TGTGGTGCCAGATCTTCTCCATGT-3. The fold changes of each target mRNA expression relative to $\beta$-actin were calculated based on the threshold cycle $\left(\mathrm{C}_{\mathrm{T}}\right)$ as $r=2^{-\Delta\left(\Delta \mathrm{C}_{\mathrm{T}}\right)}$, where $\Delta \mathrm{C}_{\mathrm{T}}=\mathrm{C}_{\mathrm{T}}$ (target) $-\mathrm{C}_{\mathrm{T}}(\beta$-actin $)$ and $\Delta\left(\Delta \mathrm{C}_{\mathrm{T}}\right)=\Delta \mathrm{C}_{\mathrm{T}}$ (experimental) $-\Delta \mathrm{C}_{\mathrm{T}}$ (control). A Bio-Rad CFX96 Real-Time System and calculations were performed using Microsoft Excel.

2.10. Cell Sheet Preparation. MSC $\left(3.2 \times 10^{5} \mathrm{cells} / \mathrm{cm}^{2}\right)$ treated with exosomes from either MSC ${ }^{\mathrm{Ctrl}}$ or $\mathrm{MSC}^{\mathrm{CR} 4}$ were seeded onto $3.5 \mathrm{~cm}$ temperature-responsive dishes purchased from CellSeed Inc. (Tokyo, Japan) and cultured cells with 15\% FBSDMEM at $37^{\circ} \mathrm{C}$. Then, confluent cells on the temperatureresponsive dishes were incubated at $20^{\circ} \mathrm{C}$. By $10-20 \mathrm{~min}$, cells detached spontaneously and floated up into the medium as monolayered cell sheet. Immediately after detachment, cell sheet was transferred onto the infarcted area of the myocardium as described $[13,14]$.

2.11. Surgical Procedures for Myocardial Infarction. Ligation of the left anterior descending artery (LAD) was performed in SD female rats (200-250 g), as previously described [12]. Briefly, isoflurane anesthesia was induced by spontaneous inhalation and maintained under general anesthesia with $1-2 \%$ isoflurane. The inhalation gas was a mixture of air and oxygen and isoflurane. The animals were mechanically ventilated using a rodent ventilator (Model 683, Harvard Apparatus, South Natick, MA) connected to a tracheal tube. Body temperature was maintained at $37^{\circ} \mathrm{C}$ throughout the surgical procedure. The heart was exposed by left side limited thoracotomy and LAD was ligated with a 6-0 polyester suture $1 \mathrm{~mm}$ from tip of the normally positioned left auricle. Before closing the thoracic cavity, positive end-expiratory pressure was applied to inflate the lungs fully; then, muscle layers and skin were closed separately. This model provides accurate and reproducible information about infarct size. To investigate the role of exosome in heart functions, SD rats were randomly divided into the following groups: (1) sham group: sham operated rats had a loose suture placed around the LAD coronary artery; (2) MI group (MI alone created by LAD ligation); (3) MI + PBS group (MI + Phosphate buffered saline alone treatment); (4) MI + MSC group (MI + ordinary MSC sheet); (5) MI + MSC ${ }^{\text {ExoCtrl }}$ group (MI + exosomes isolated from MSC transfected with null lentivirus vector, then treated with MSC sheet); (6) MI + MSC ${ }^{\text {ExoCR4 }}$ group (MI + exosomes isolated from MSC transfected with CXCR4 over-expressing lentivirus vector and then treated with MSC sheet); (7) MI $+\mathrm{MSC}^{\text {ExosiCR4 }}$ group (MI + exosomes isolated from MSC transfected with siRNA against CXCR4 lentivirus vector and then treated with MSC sheet). 
2.12. Immunohistochemical Analysis. The vascularization was identified by von Willebrand factor (vWF) expression [12]. Briefly, heart tissues at 4 weeks after MI were harvested, fixed in $10 \%$ formalin, and sectioned at $5 \mu \mathrm{m}$ thickness. Cardiac troponin T (cTnT) was used to identify CM. Signals were visualized with secondary antibodies conjugated to donkey anti-rabbit (FITC; Jackson ImmunoResearch) or donkey anti-mouse (Tritc; Jackson ImmunoResearch), respectively. DAPI was used to identify nuclei. Fluorescent imaging was performed with Olympus BX41 microscope and vessel numbers per $\mathrm{mm}^{2}$ were counted in 4 randomly selected fields.

2.13. Analysis of Left Ventricular (LV) Fibrosis. Fixed heart tissues were embedded in paraffin, and LV cross-sections from mid-LV to apex stained with Masson trichrome were used to quantify fibrosis in the left ventricle in various treatment groups. The LV area images on each slide were filmed by using an Olympus BX41 microscope equipped with CCD (MagnaFire, Olympus) camera. LV fibrosis area and total LV area of each image were measured using the ImagePro Plus (Media Cybernetics Inc., Carlsbad, CA, USA), and the fibrosis area as a percentage of the total LV area was calculated as (fibrosis area/total LV area) $\times 100 \%$.

2.14. Cardiac Function Assessment by Echocardiography. Echocardiography (iE33 Ultrasound System, Phillips) with a $15 \mathrm{MHz}$ probe was performed 4 weeks after cell sheet placement, blinded to the study group or treatment, to assess systolic, diastolic dimensions. Hearts were imaged in 2D long-axis view at the level of the greatest LV diameter with animals under light general anesthesia. LV end-diastolic and end-systolic diameters were measured from $\mathrm{M}$-mode recordings using the leading-edge method. LV ejection fraction (EF) was calculated as $\mathrm{EF}(\%)=$ [left ventricular end-diastolic dimension $(\mathrm{LVDd})^{3}-$ left ventricular end systolic dimension $\left.(\mathrm{LVDs})^{3}\right] /(\mathrm{LVDd})^{3} \times 100 \%$. Fractional shortening $(\mathrm{FS})$ at the level of the MI and patch application was also determined as [(LVDd - LVDs)/LVDd] $\times 100 \%$. All measurements were performed according to the American Society for Echocardiography leading-edge technique and averaged over three consecutive cardiac cycles.

2.15. Statistical Analysis. Results were statistically analyzed with the use of the StatView 5.0 software package (Abacus Concepts Inc., Berkeley, CA). All values were expressed as mean \pm SEM. Comparison between groups for echocardiographic longitudinal data was performed by two-way analysis of variance (ANOVA) for repeated measures. All resulting differences were analyzed by one-way ANOVA, followed by a Bonferroni/Dunn test or unpaired $t$-test. $P$ value $<0.05$ was considered statistically significant.

\section{Results}

3.1. Isolation and Identification of the MSC-Secreted Exosomes. Putative exosome fractions from conditioned media of MSC were isolated to investigate the paracrine effect of MSC via exosome release. High resolution transmission electron micrographs showed that MSC-derived exosomes exhibited rounded and double-membrane structures with a size of approximately $40-90 \mathrm{~nm}$ (Figure $1(\mathrm{a})$ ), which is similar to previous descriptions $[6,7]$. DLS analysis was performed to define the size of these exosomes. The size distribution profile displayed a bell-shaped curve, suggesting a physically homogeneous population with a peak at $90 \mathrm{~nm}$ (Figure 1(b)). Western blot analysis confirmed that exosome fraction expressed CD9 and CD63, both well-established exosome markers [4] (Figure 1(c)). In contrast, the exosome release from MSC was blocked by sphingomyelinase inhibitor GW4869 pretreatment, as indicated by significant downregulation of CD9 and CD63 expression levels. Exosomes were directly labeled with PKH67 (green fluorescence) and then cocultured with CM to determine whether the secreted exosomes were incorporated into recipient cells. Immunocytochemical analysis demonstrated that PKH67 (green color) was colocalized in the most sarcomeric $\alpha$-actinin positive CM (red color) after $48 \mathrm{hr}$. of coculture (Figure 1(d)).

3.2. MSC Released CXCR4-Enriched Exosomes. In order to assess whether MSC-derived exosomes possess CXCR4 and provide an alternative CXCR4 protein delivery system, lentivirus conveying control vector, CXCR4 overexpressing vector, and CXCR4-siRNA were constructed as confirmed by Western blotting (Figure 2(a)). Exosomes were then isolated from cell culture mediums of various MSC lines to identify the cargo loading CXCR4. Western blots revealed that the exosomes derived from $\mathrm{MSC}^{\mathrm{CR} 4}$ (Exo ${ }^{\mathrm{CR} 4}$ ) exhibited a significantly higher CXCR4 level than MSC ${ }^{\mathrm{Ctrl}}$-derived exosomes (Exo $^{\mathrm{Ctrl}}$ ) (Figure 2(b)). However, with siRNA against CXCR4 in MSC (MSC ${ }^{\mathrm{siCR} 4}$ ), the CXCR4 level was significantly downregulated, leading to a significant decrease CXCR4 level in exosomes (Exo ${ }^{\text {siCR4 }}$ ) as compared with other groups (Figure 2(b)).

3.3. The Antiapoptosis Effect of CXCR4-Enriched Exosomes on CM via Activating PI3K/Akt-Associated Signaling Pathways. In order to assess the functional transfer of CXCR4 by exosomes and the potential to correct the various hypoxiainduced cascades and prevent occurrence of apoptosis, rat $\mathrm{CM}$ with exosomes derived from $\mathrm{MSC}^{\mathrm{Ctrl}}$, $\mathrm{MSC}^{\mathrm{CR} 4}$, or MSC $^{\text {siCR4 }}$ were treated in hypoxic conditions to explore whether exosomes facilitate the uptake of therapeutic proteins into injured cells that contribute to cardiac protection. TUNEL analysis showed the number of apoptotic cells (green) was significantly decreased in Exo ${ }^{\mathrm{CR} 4}$ treated CM $\left(\mathrm{CM}+\mathrm{Exo}^{\mathrm{CR} 4}\right.$ group, $\left.21.3 \% \pm 1.3\right)$ as compared to Exo ${ }^{\mathrm{Ctrl}}$ treated CM $\left(\mathrm{CM}+\right.$ Exo $^{\mathrm{CR} 4}$ group, $53.6 \% \pm 2.7$; Figures 3(a) and $3(\mathrm{~b}))$. The antiapoptotic effects of CXCR 4 were abolished, as evidenced by increased TUNEL positive CM number in $\mathrm{CM}+\mathrm{Exo}^{\mathrm{CR} 4}+\mathrm{LY}$ (PI3K inhibitor LY294002) group as compared with $\mathrm{CM}+\mathrm{Exo}^{\mathrm{CR} 4}$ group (Figure 3). The effects of CXCR4-enriched exosomes were completely abolished by siRNA targeting CXCR4 (62.4\% \pm 2.9 , Figures 3(a) and 3(b)). The IGF-1 $\alpha / \mathrm{PI} 3 \mathrm{~K} /$ Akt pathway was assessed to elucidate the 


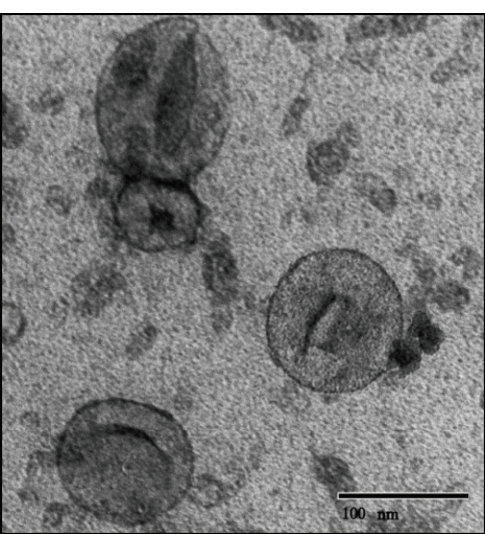

(a)

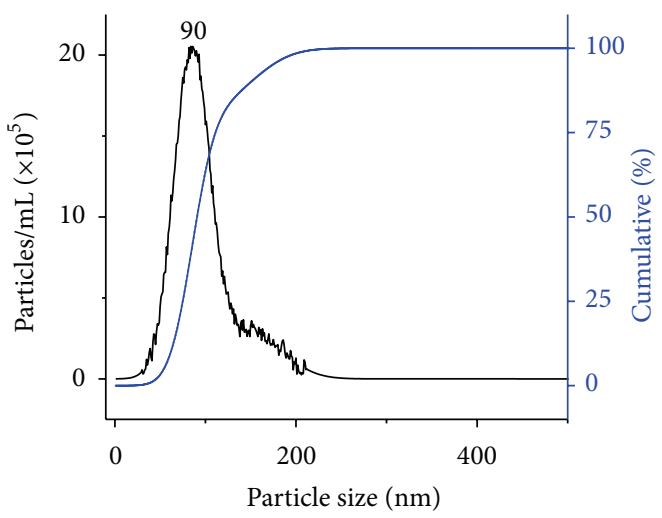

(b)

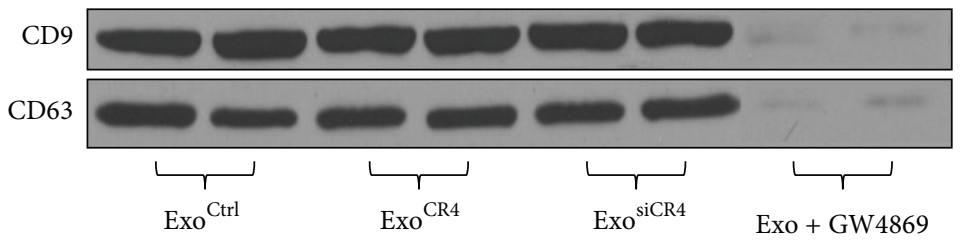

(c)

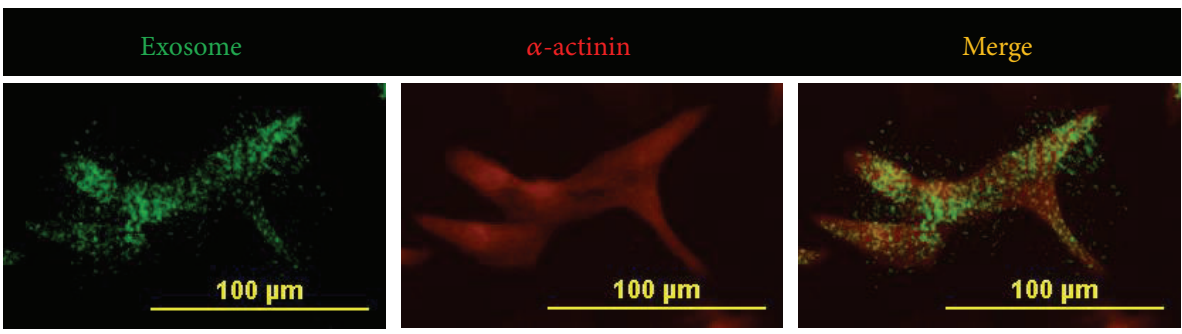

(d)

FIGURE 1: Characterization of MSC-derived exosomes. (a) Transmission electron microscopy (TEM) micrograph of exosomes purified from MSC. Isolated exosomes showed spherical and membrane encapsulated with the diameters varying between $40 \mathrm{and} 90 \mathrm{~nm}$. (b) Representative dynamic light scattering (DLS) number distribution measurement of isolated exosome population demonstrates a single peak ( $90 \mathrm{~nm}$ diameter) indicating they are free of contamination. (c) Expression of specific exosomal markers, CD9 and CD63, in exosomes was identified by Western blotting. Exo ${ }^{\mathrm{Ctrl}}$, exosomes isolated from MSC transfected with null lentivirus (MSC ${ }^{\mathrm{Ctrl}}$ ); Exo ${ }^{\mathrm{CR} 4}$, exosomes isolated from MSC transfected with lentivirus overexpressing CXCR4 $\left(\mathrm{MSC}^{\mathrm{CR} 4}\right)$; Exo ${ }^{\text {siCR4 }}$, exosomes isolated from MSC transfected with lentivirus siRNA against CXCR4 gene (MSC ${ }^{\text {siCR4 }}$ ); Exo + GW4860, exosomes isolated from MSC with additional sphingomyelinase inhibitor GW4860. (d) Uptake of exosomes by neonatal cardiomyocytes (CM). CM were stained by cardiac specific antibody, sarcomeric $\alpha$-actinin (red). MSC ${ }^{\mathrm{CR} 4}$-derived exosomes were labeled with PKH67 (green) and incubated with CM for $48 \mathrm{hr}$.

potential mechanisms responsible for enhanced survival rate of Exo ${ }^{\mathrm{CR} 4}$ treated CM under hypoxic conditions.

IGF- $1 \alpha$ and phosphorylation of Akt (pAkt) as well as procaspase 3 levels dramatically increased in $\mathrm{CM}+\mathrm{Exo}^{\mathrm{CR} 4}$ group as compared to $\mathrm{CM}+\mathrm{Exo}^{\mathrm{Ctrl}}$ group and $\mathrm{CM}+\mathrm{Exo}^{\text {siCR4 }}$ group, whereas the level of active caspase 3 was significantly downregulated (Figure 4(a)). In contrast, with the selective PI3K inhibitor LY294002 (LY), activated PI3K/Akt pathway induced by Exo ${ }^{\mathrm{CR} 4}$ was inhibited as indicated by significantly decreased expression of pAkt and procaspase 3 and increased expression of active caspase 3 , as compared to $\mathrm{CM}+$ Exo $^{\mathrm{CR} 4}$ (Figures 4(c), 4(d), and 4(e)). However, no significant difference was observed among $\mathrm{CM}+\mathrm{Exo}^{\mathrm{Ctrl}}, \mathrm{CM}+\mathrm{Exo}^{\text {siCR4 }}, \mathrm{CM}$
$+\mathrm{Exo}^{\mathrm{Ctrl}}+\mathrm{LY}$, and CM + Exo ${ }^{\mathrm{CR} 4}+$ LY groups. Interestingly, LY294002 had no effect on IGF-1 $\alpha$ expression (Figure 4(b)).

3.4. Effect of Exo ${ }^{C R 4}$ on Tube Formation in HUVEC. Tube formation assay was performed using HUVEC to evaluate proangiogenic effects of CXCR4-enriched exosomes in vitro. Specifically, HUVEC in the control treatment group $\left(\right.$ HUVEC + Exo $\left.{ }^{\mathrm{Ctrl}}\right)$ exhibited small round shapes and did not spread. However, when HUVEC were treated with Exo ${ }^{\mathrm{CR} 4}$ for $16 \mathrm{hr}$., they started migration and alignment, followed by the development of capillary tubes, sprouting of new capillaries, and finally formation of the cellular networks (Figures 5(a) and 5(b)). These cells became elongated, forming thing cords 

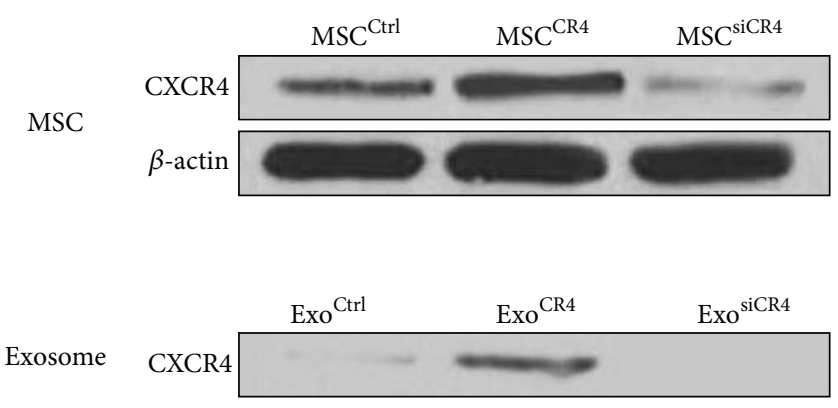

(a)

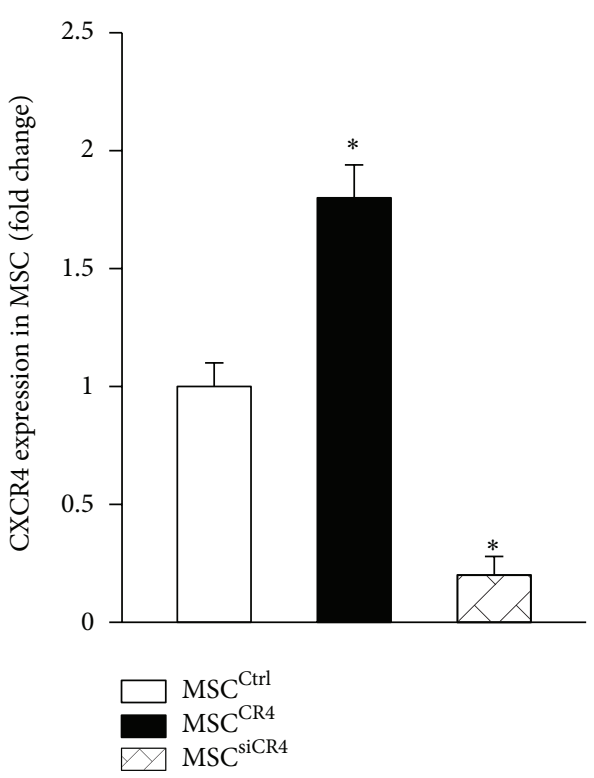

(b)

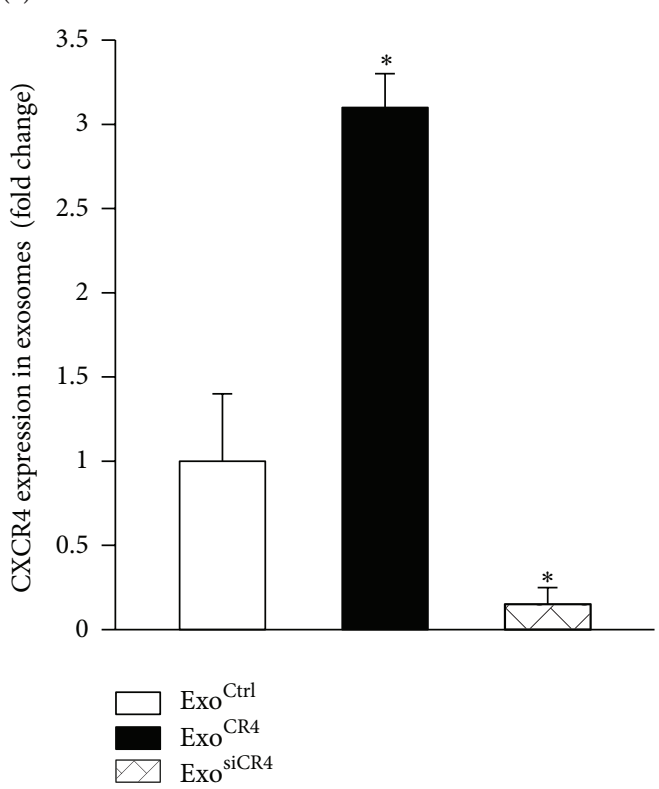

(c)

FIGURE 2: Expression of CXCR4 in MSC and MSC-derived exosomes. (a) CXCR4 expression was identified by Western blotting in MSC from various groups with quantitative analysis. ((b) and (c)) The expression of CXCR4 in MSC-derived exosome was detected by Western blotting and quantitative analysis. All values expressed as mean \pm SEM. $^{*} P<0.05$ versus MSC $^{\text {ctrl }}$ or Exo ${ }^{\mathrm{Ctrl}}, n=4$ for each group.

of interconnecting cells, and branching to form a network of capillary-like structures. The number of tube-like structures was significantly increased in $\mathrm{HUVEC}+\mathrm{Exo}^{\mathrm{CR} 4}$ group as compared to HUVEC + Exo $^{\text {Ctrl }}$ or HUVEC + Exo $^{\text {siCR4 }}$ group, or any other groups (Figure 5(b)), accompanied with significant upregulation of VEGF level (Figure 5(c)). However, the proangiogenic effect of Exo ${ }^{\mathrm{CR} 4}$ was attenuated by LY294002 treatment $\left(\mathrm{HUVEC}+\mathrm{Exo}^{\mathrm{CR} 4}+\mathrm{LY}\right)$ as indicated by downregulation of VEGF level as well as significantly reduced tube-like structure number as compared to Exo ${ }^{\mathrm{CR} 4}$ treated group.

3.5. Exo ${ }^{C R 4}$ Promotes Angiogenesis and Reduces Infarction Size. To verify whether CXCR4-enriched exosomes play a role in angiogenesis for the repair of infarcted heart in vivo, we implanted cell patch system pretreated with exosomes from various groups. At 4 weeks after exosome pretreated cell patch graft, we assessed angiogenesis in the infarcted heart using immunostaining for von Willebrand factor (vWF)
(Figure 6(a)). Quantitative data showed that the number of vWF positive vessels was markedly increased in Exo ${ }^{\mathrm{CR} 4}$ pretreated group $\left(\mathrm{MI}+\mathrm{MSC}^{\text {ExoCR4 }}\right)$ as compared to control group (Figure 6(b)). Additionally, the infarction size was significantly reduced in $\mathrm{MI}+\mathrm{MSC}, \mathrm{MI}+\mathrm{MSC}^{\text {ExoCtrl }}, \mathrm{MI}+$ $\mathrm{MSC}^{\text {ExosiCR4 }}$, and MI + MSC ${ }^{\text {ExoCR4 }}$ groups as compared to MI group or $\mathrm{MI}+\mathrm{PBS}$ group, but $\mathrm{MI}+\mathrm{MSC}^{\text {ExoCR4 }}$ group was the lowest as assessed by Masson's trichrome staining (Figures $6(\mathrm{c})$ and $6(\mathrm{~d}))$.

3.6. Assessment of Cardiac Functions. In order to gain an understanding of whether MSC sheet pretreated with CXCR4-enriched exosomes contributes to functional recovery of ischemic heart in vivo, cardiac functions (LVDd, LVDs, EF, and FS) were evaluated by echocardiography. Although the LV cavity (LVDd and LVDs) was dilated 4 weeks after cell sheet implantation after MI (Figure 7(a)) in comparison to sham group, the $\mathrm{MI}+\mathrm{MSC}$ or $\mathrm{MI}+\mathrm{MSC}^{\text {ExoCtrl }}$ group, or $\mathrm{MI}+\mathrm{MSC}^{\text {ExosiCR4 }}$ group, or $\mathrm{MI}+\mathrm{MSC}^{\text {ExoCR4 }}$ group 


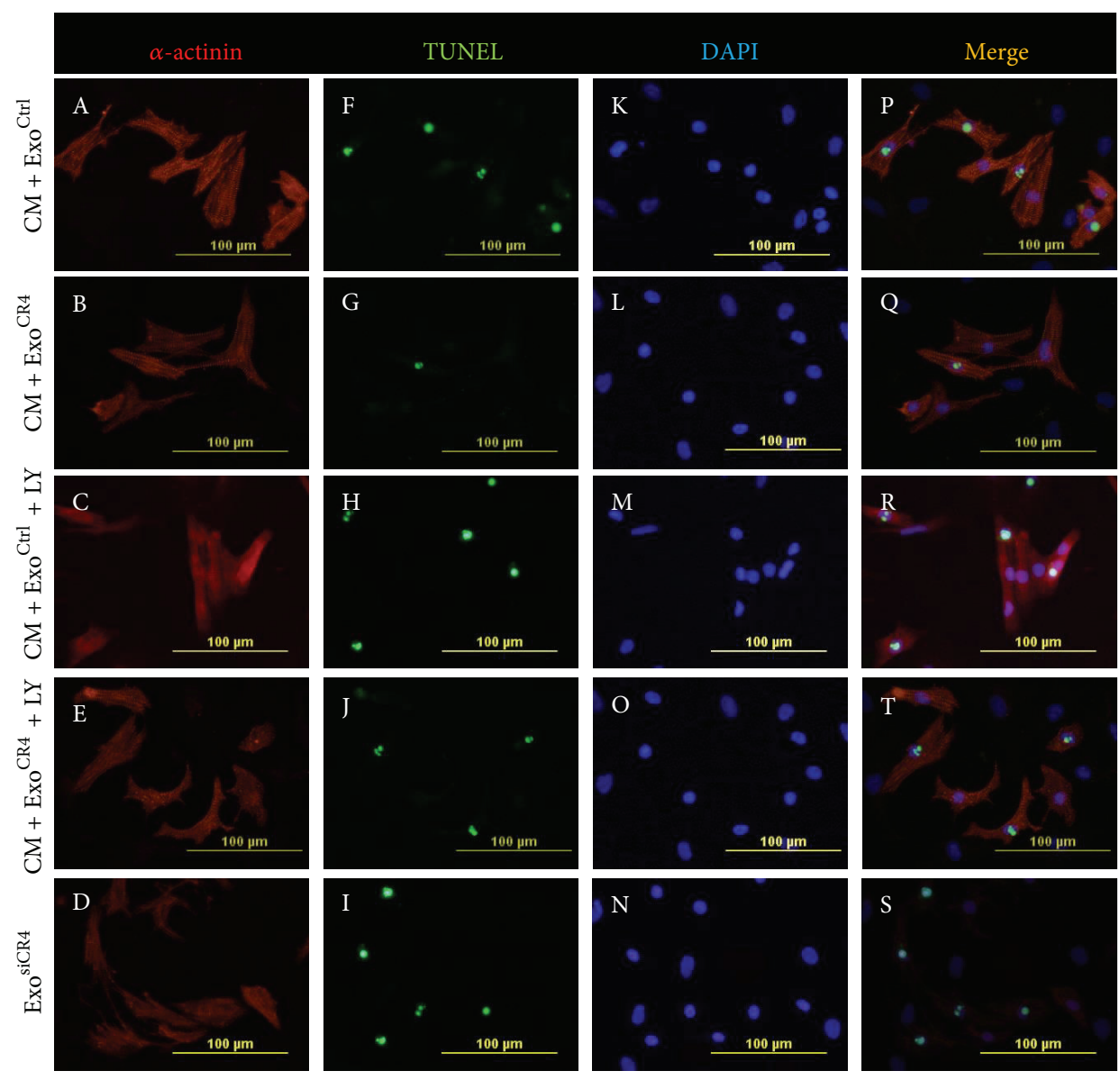

(a)

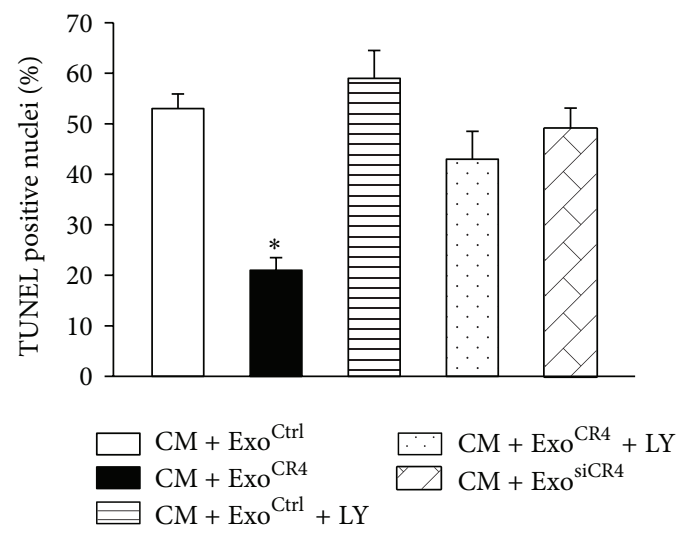

(b)

FIgURE 3: The effect of MSC ${ }^{\mathrm{CR} 4}$-derived exosomes on apoptosis. (a) TUNEL staining for CM apoptosis under hypoxic condition. CM labeled with cardiac specific antibody, sarcomeric $\alpha$-actinin (red). Nuclei were labeled with DAPI (blue) and TUNEL positive nuclei (green). Original magnification $\times 200$. (b) Quantitative data for the numbers of TUNEL positive nuclei in various experimental groups. All values expressed as mean \pm SEM. $^{*} P<0.05$ versus Exo ${ }^{\mathrm{Ctrl}}, n=6$ for each group. $\mathrm{CM}+\mathrm{Exo}^{\mathrm{Ctrl}}, \mathrm{CM}$ treated with MSC ${ }^{\mathrm{Ctrl}}$-derived exosomes; CM + Exo ${ }^{\mathrm{CR} 4}, \mathrm{CM}$ treated with MSC $^{\mathrm{CR} 4}$-derived exosomes; CM + Exo ${ }^{\mathrm{Ctrl}}+\mathrm{LY}, \mathrm{CM}+\mathrm{Exo}^{\mathrm{Ctrl}}$-treated with LY294002 (a PI3K inhibitor); CM + Exo ${ }^{\mathrm{CR} 4}+\mathrm{LY}, \mathrm{CM}$ $+\mathrm{Exo}^{\mathrm{CR} 4}$ treated with LY294002; CM + Exo ${ }^{\text {siCR4 }}, \mathrm{CM}$ treated with $\mathrm{MSC}^{\text {siCR4 }}$-derived exosomes. 


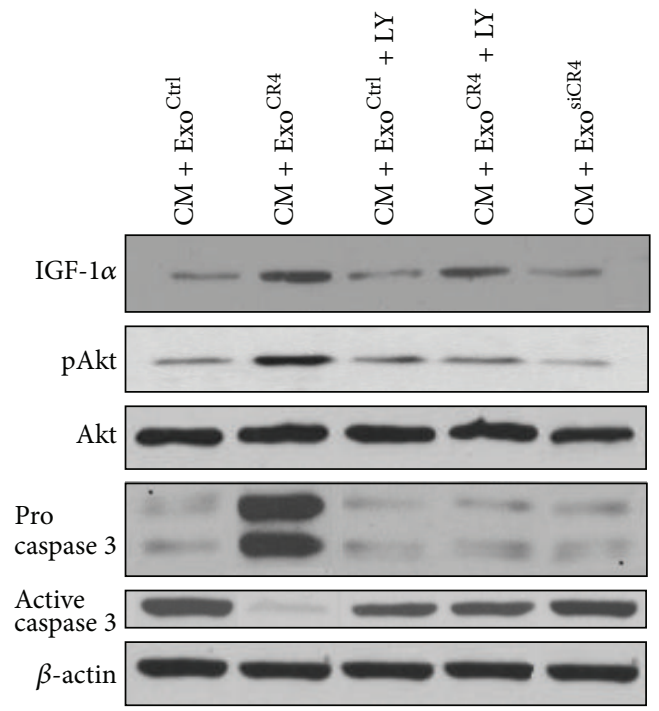

(a)

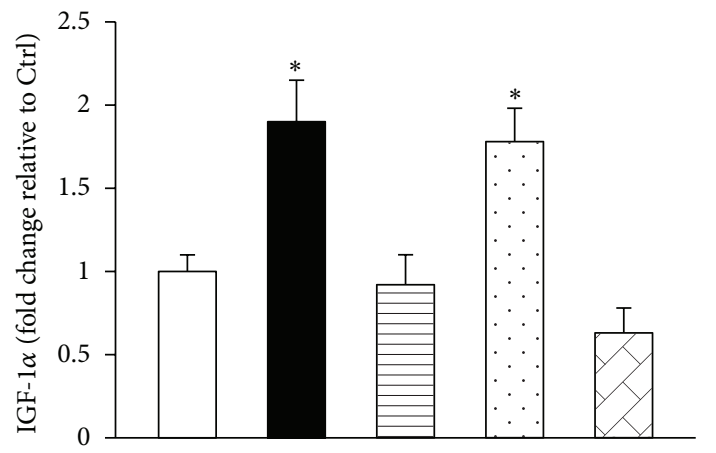

(b)

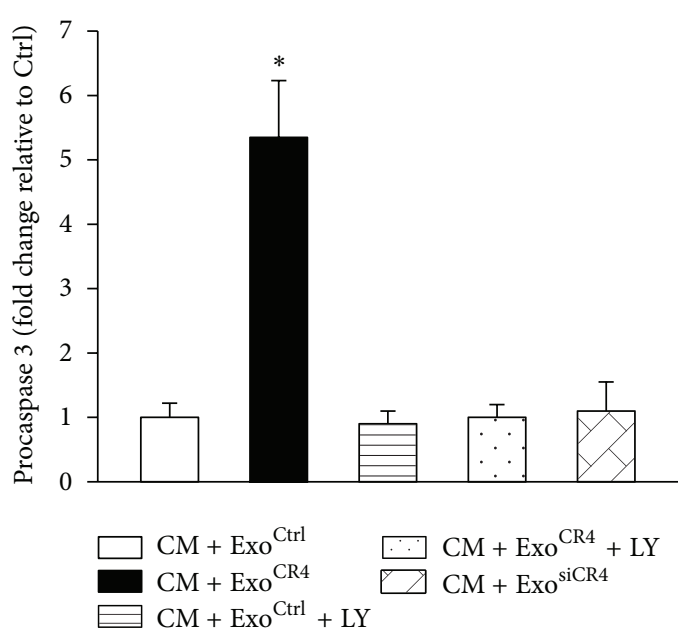

(d)

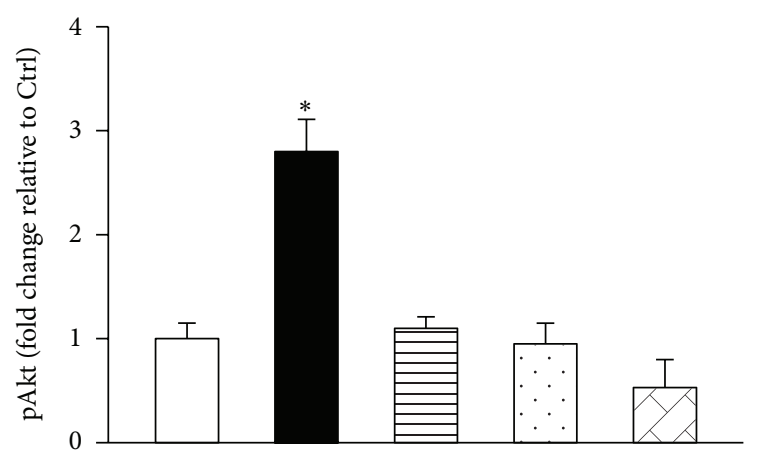

(c)

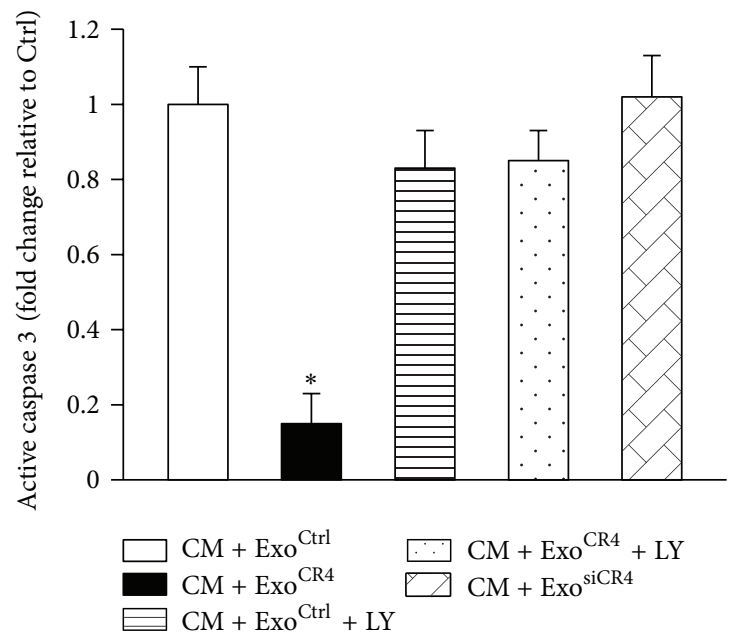

(e)

FIGURE 4: The expression of Akt signaling pathway related genes in CM with various exosome treatments. (a) The expression level of pAkt, IGF-l $\alpha$, procaspase 3, and active caspase 3 in CM was analyzed by Western blotting. ((b)-(e)) Quantitative data of pAkt, IGF-l $\alpha$ procaspase 3 , and active caspase 3 in $C M .{ }^{*} P<0.05$ versus $\mathrm{CM}+\mathrm{Exo}^{\mathrm{ctrl}}$. All values expressed as mean $\pm \mathrm{SEM}, n=6$ for each group. 

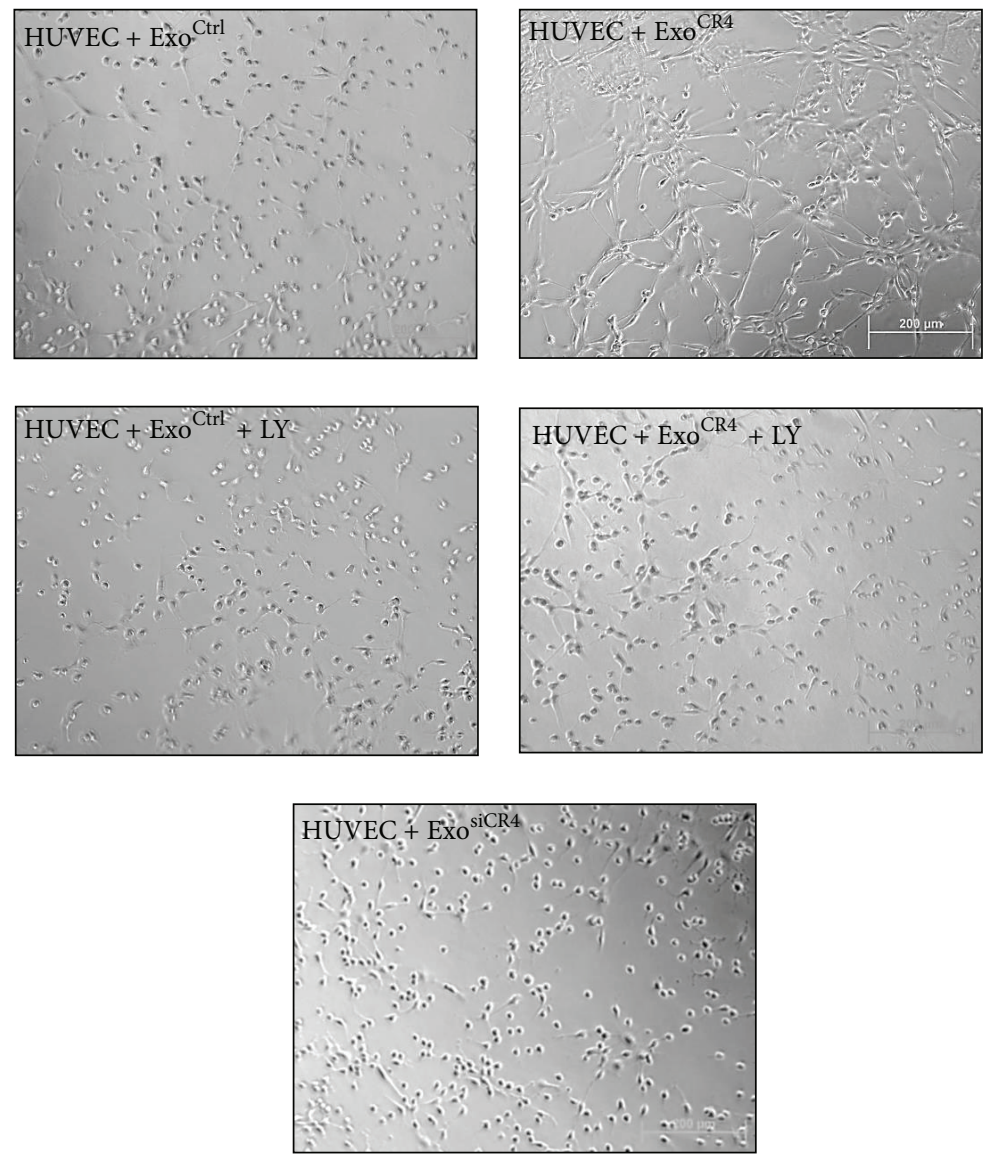

(a)
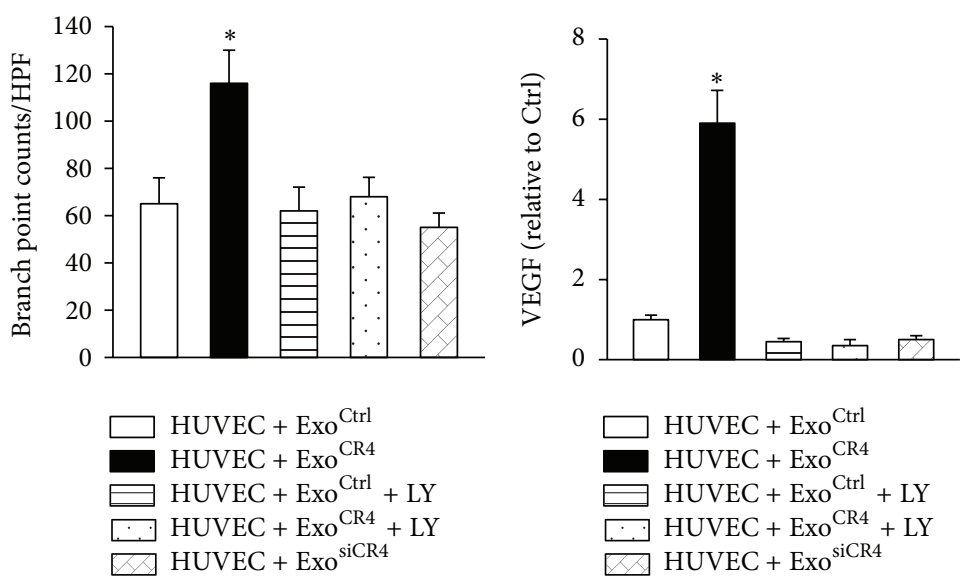

(b)

(c)

FIGURE 5: The in vitro angiogenic properties of MSC $^{\mathrm{CR} 4}$-derived exosomes. (a) Induction of tube formation by MSC-derived exosomes in HUVEC. (b) Quantitative data of total branch points in various groups. (c) VEGF expression in various groups was analyzed by qPCR. ${ }^{*} P<0.05$ versus Exo ${ }^{\text {Ctrl }}$. All values expressed as mean \pm SEM, $n=6$ for each group. HUVEC + Exo ${ }^{\text {Ctrl }}$, HUVEC treated with MSC ${ }^{\text {Ctrl }}$ derived exosomes; HUVEC + Exo ${ }^{\mathrm{CR} 4}$, HUVEC treated with MSC $^{\mathrm{CR} 4}$-derived exosomes; HUVEC + Exo ${ }^{\mathrm{Ctrl}}+\mathrm{LY}_{\text {, HUVEC + Exo }}{ }^{\mathrm{Ctrl}}$ treated $^{\text {to }}$ with LY294002 (a PI3K inhibitor); HUVEC + Exo ${ }^{\mathrm{CR} 4}+\mathrm{LY}, \mathrm{HUVEC}+\mathrm{ExO}^{\mathrm{CR} 4}$ treated with LY294002; HUVEC + Exo ${ }^{\text {siCR4 }}$, HUVEC treated with MSC $\mathrm{MiCR}^{\text {sic }}$-derived exosomes. 


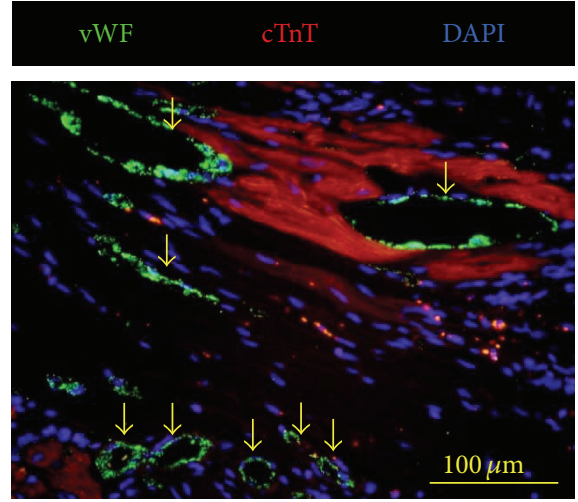

$\mathrm{MI}+\mathrm{MSC}^{\mathrm{ExoCtrl}}$

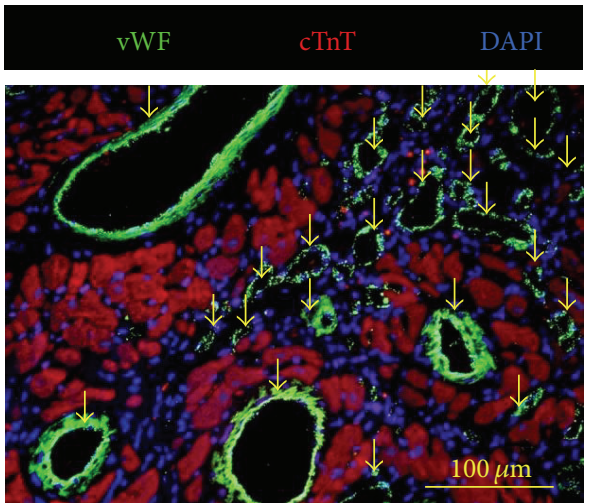

$\mathrm{MI}+\mathrm{MSC}^{\mathrm{ExoCR} 4}$

(a)

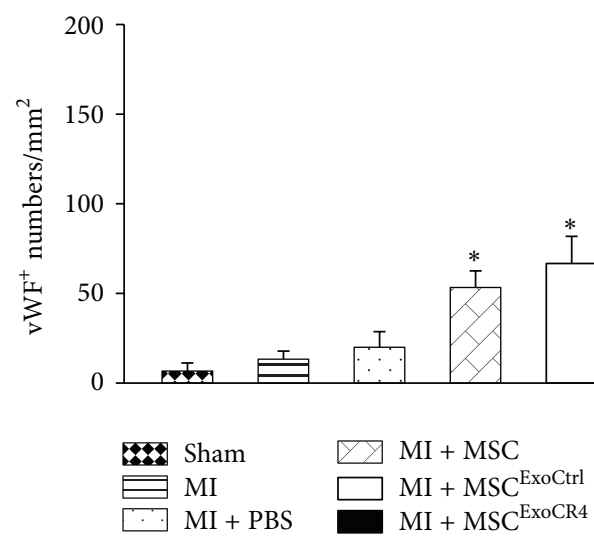

(b)

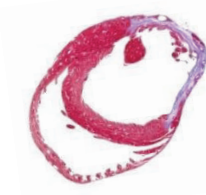

$\mathrm{MI}+\mathrm{MSC}^{\text {ExoCtrl }}$

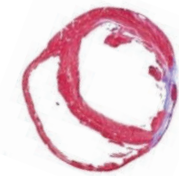

$\mathrm{MI}+\mathrm{MSC}^{\mathrm{ExoCR}}$

(c)

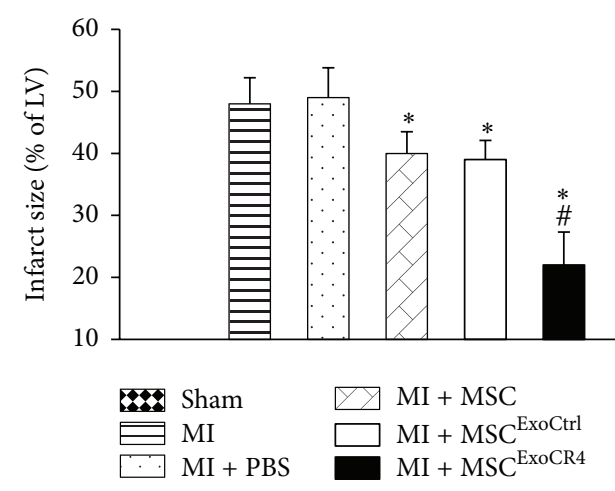

(d)

FIGURE 6: Effects of exosome pretreated MSC patch on in vivo angiogenesis in infarcted myocardium. (a) The vessel formation in the infarcted border area was identified by vWF (green color, yellow arrows). Cardiomyocytes were identified by cardiac troponin T (cTnT) and all nuclei were stained with DAPI. Scale bar $=100 \mu \mathrm{m}$. Original magnification $\times 400$. (b) Quantitative data for vessel density in treatment groups. ${ }^{*} P<0.05$ versus MI group; ${ }^{\#} P<0.05$ versus MI + MSC $^{\text {ExoCtrl }}$ group. (c) Masson's trichrome staining and quantitative data for fibrotic size in various treatments. (d) The percentage of infarct size in hearts of various groups. ${ }^{*} P<0.05$ versus MI; ${ }^{\#} P<0.05$ versus MI + MSC ${ }^{\text {ExoCtrl }}$ group. Sham: sham operated rats had a loose suture placed around the LAD coronary artery; MI (myocardial infarction alone created by LAD

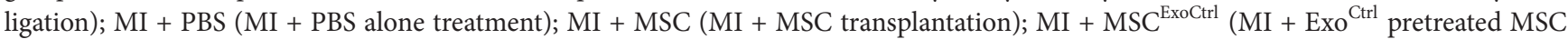
transplantation); MI + MSC ${ }^{\text {ExosiCR4 }}\left(\mathrm{MI}+\right.$ Exo $^{\text {siCR4 }}$ pretreated MSC); MI + $\mathrm{MSC}^{\mathrm{ExoCR} 4}\left(\mathrm{MI}+\mathrm{Exo}^{\mathrm{CR} 4}\right.$ pretreated MSC). $^{*} \mathrm{P}<0.05$ versus MI; ${ }^{\#} P<0.05$ versus MI + MSC. All values expressed as mean $\pm \mathrm{SEM}, n=6$ for each group. 


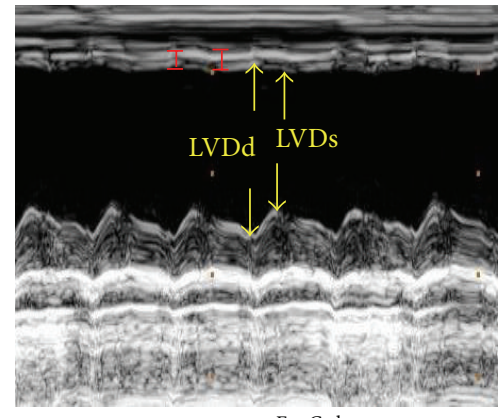

$\mathrm{MI}+\mathrm{MSC}^{\mathrm{ExoCtrl}}$

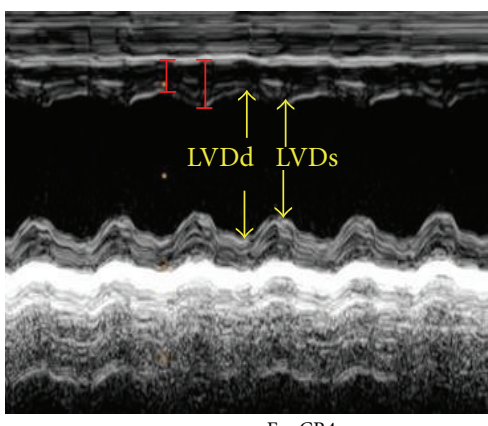

$\mathrm{MI}+\mathrm{MSC}^{\mathrm{ExoCR} 4}$

(a)

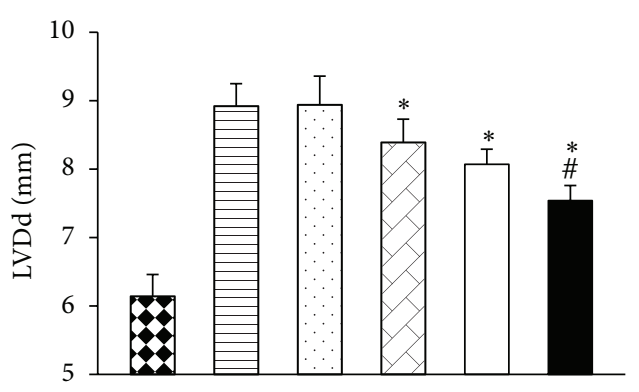

(b)

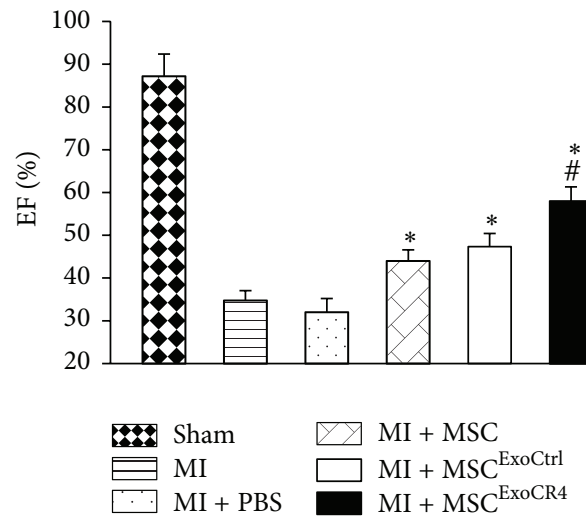

(d)

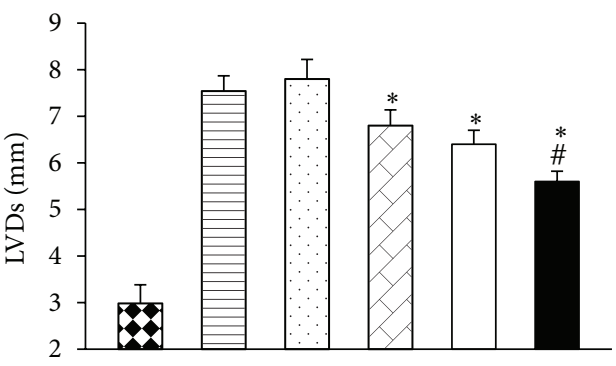

(c)

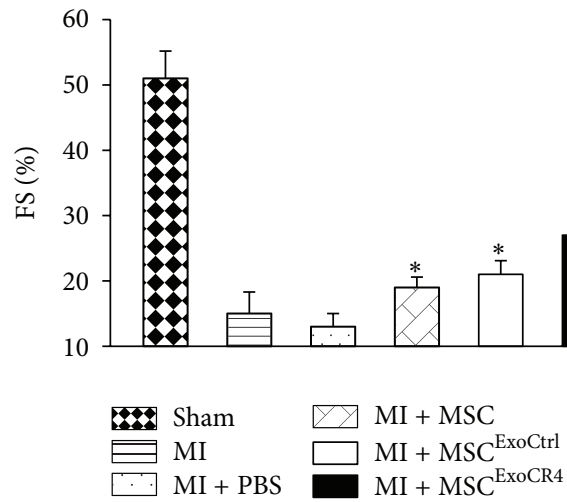

(e)

Figure 7: Cardiac function assessed by echocardiography at 4 weeks after cell patch graft. (a) M-mode echocardiography data in various experimental groups. ((b)-(e)) Quantification analysis for LVDd, LVDs, EF, and FS. LVDd: left ventricular diastolic left ventricular diameter, LVDs: systolic left ventricular diameter, EF: left ventricular ejection fraction, and FS: left ventricular fraction shortening. ${ }^{\#} P<0.05$ versus MI + MSC $^{\text {ExoCtrl }}$ group. ${ }^{*} P<0.05$ versus MI; ${ }^{\#} P<0.05$ versus MI + MSC. All values expressed as mean \pm SEM, $n=6$ for each group.

exhibited a significantly reduced $\mathrm{LV}$ remodeling, and $\mathrm{MI}+$ MSC $^{\text {ExoCR4 }}$ group exhibited the most significantly reduced $\mathrm{LV}$ remodeling as compared to $\mathrm{MI}$ or $\mathrm{MI}+\mathrm{PBS}$ group, as shown by decreased LVDd and LVDs (Figures 7(b) and 7(c)). Furthermore, EF and FS were significantly higher in MI + MSC and MI + MSC $^{\text {ExoCtrl }}$ groups, but most significantly increased in the MI + MSC ${ }^{\text {ExoCR4 }}$ group as compared to MI or MI + PBS group (Figures 7(d) and 7(e)).

\section{Discussion}

Cell-based therapy has emerged as a promising strategy for the treatment of ischemic heart diseases via myocardial repair. The paracrine effects of exosomes introduce an alternative solution to the therapeutic applications of MSC in regenerative medicine $[4,15]$. Indeed, exosomes can achieve some beneficial effects through the usage of small soluble biological compounds such as growth factors, chemokines, cytokines, transcription factors, genes, and RNAs [15]. However, the delivery of these factors to the target cells with stable integrity and biological potency remains a challenge. As a bilipid membrane vesicle, exosomes not only have the capacity to carry a large cargo load, but also protect the contents such as protein and RNA from degradation by enzymes or chemicals $[14,15]$. In recent years, exosomes have also been implicated in cell communication or pathogenesis. For example, exosomes secreted by cardiomyocyte progenitor 


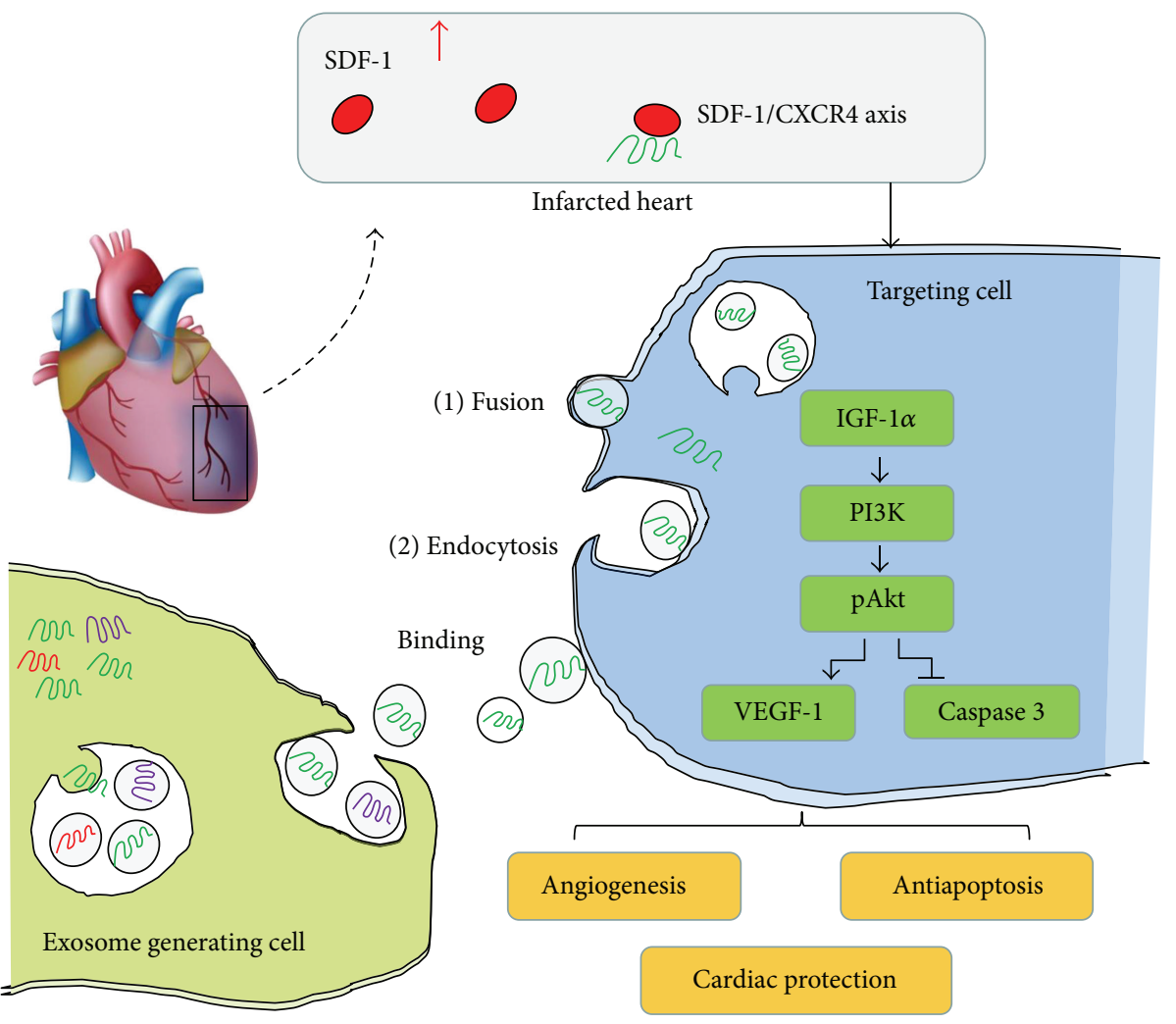

FIGURE 8: Schematic depiction of functional exosomes secreted from MSC overexpressing CXCR4 for activation of signaling pathways for restoration of LV function after MI. In exosome generating cells, CXCR4 are selectively incorporated into the intraluminal vesicles (IVLs) of multivesicular endosomes (MVEs) from the plasma membrane. Then, by fusing with the plasma membrane, MVEs release CXCR4enriched exosome into the extracellular milieu. CXCR4-enriched exosomes can bind to the plasma membrane of the targeting cells. These recruited CXCR4-enriched exosomes may either fuse directly with the plasma membrane or fuse with delimiting membrane of an endocytic compartment. Then, released CXCR 4 can be delivered into the membrane or cytosol of the targeting cell which contributes to SDF-1 $\alpha /$ CXCR 4 interaction. In infarcted heart tissue, increased binding of SDF-1 $\alpha$ to CXCR4 activates G-protein-coupled receptor kinases, which activate a cascade of signaling pathways in cells. Pretreatment of stem/progenitor cells with CXCR4-enriched exosome can reduce MI-induced cell death and promote angiogenesis through PI3K/Akt signaling pathway activation, thereby enhancing heart function improvement.

cells were reported to stimulate the migration of the endothelial cells [16].

The potential role of CXCR4-enriched exosomes was demonstrated for the repair of the infarcted heart (Figure 8). The in vitro studies showed that CXCR4-enriched exosomes released by $\mathrm{MSC}^{\mathrm{CR} 4}$ were transferred to cardiomyocytes, leading to an increase in cardiomyocyte survival under hypoxic conditions. This was also associated PI3K/Akt signaling pathway activation. In vivo studies demonstrated that cell sheets pretreated with CXCR4-enriched exosomes promoted angiogenesis and demonstrated subsequent cardiac function improvement. Evidence was also provided showing that MSC ${ }^{\mathrm{CR} 4}$ enhances the release of proangiogenic factors and promotes MSC endothelial differentiation under hypoxic conditions and that cardiac functions improved when MSC ${ }^{\mathrm{CR} 4}$ cell patches were implanted into myocardial ischemic hearts [17]. A study by Lai et al. also showed that exosomes secreted by MSC were able to reduce myocardial ischemia/reperfusion injury [18], which supports the results of this study. Purified exosomes from bone marrow-derived MSC were isolated using differential centrifugation and
ExoQuick precipitation to investigate the mechanisms of paracrine activities of exosomes from CXCR4 overexpressing MSC. It was found that MSC-derived exosomes had phenotypes similar to those derived from other sources $[7,14]$. Interestingly, the expression level of CXCR4 was significantly higher both in MSC ${ }^{\mathrm{CR} 4}$ and Exo ${ }^{\mathrm{CR} 4}$ over those of MSC ${ }^{\mathrm{Ctrl}}$ and MSC $^{\text {siCR4 }}$, suggesting that the level of CXCR4 in exosomes can be enhanced by overexpressing CXCR4 in MSC.

CXCR4 is a G-protein-coupled receptor and can activate several G-protein-mediated downstream signaling pathways after stimulation [2, 19]. This is a critical factor involved in homing, endothelial cell migration, and engraftment of hematopoietic stem and progenitor cells [2]. One endpoint of CXCR4 signaling is the activation of transcription factors [19]. In addition, the SDF- $1 \alpha /$ CXCR 4 axis was reported to be critical for activation of $\mathrm{PI} 3 \mathrm{~K}$ in ischemic cardiomyocytes, thereby mediating acute cardioprotection [19]. Furthermore, TUNEL assay revealed that apoptosis in cardiomyocytes treated with $\mathrm{MSC}^{\mathrm{CR} 4}$-derived exosomes was significantly decreased as compared to control group, whereas this protective effect was abolished by PI3K/Akt inhibitor, LY294002, 
which led us to hypothesize that $\mathrm{MSC}^{\mathrm{CR} 4}$-derived exosomes could promote cardioprotection through the Akt signaling pathway. Expression of key downstream molecules of the Akt signaling pathway was examined by Western blot to test this hypothesis. VEGF signaling pathway plays an essential role in the vascular homeostasis and the angiogenic cascade [20]. IGF- $1 \alpha$ itself is a somewhat less efficacious inducer of angiogenesis as compared with other angiogenic growth factors such as VEGF. The antiapoptosis activity of IGF- $1 \alpha$ has been attributed to downregulation of cleaved caspase3 [21]. Caspase 3 is a member of the cysteine-aspartic acid protease (caspase) family and interacts with caspase 8 and caspase 9. Sequential activation of caspase plays a central role in the execution phase of cell apoptosis [22]. It is well proved that $\mathrm{MSC}^{\mathrm{CR} 4}$-derived exosomes activate a series of downstream growth factors, such as IGF-1 $\alpha$ and Akt. Cells were treated with LY294002, a PI3k/Akt inhibitor, to determine paracrine effects of $\mathrm{MSC}^{\mathrm{CR} 4}$-derived exosomes which were mediated through Akt signaling. Although LY294002 had no effect on IGF-1 $\alpha$ expression, the antiapoptosis effect as well as tube formation induced from $\mathrm{MSC}^{\mathrm{CR} 4}$-derived exosomes was markedly abolished. These results provided further evidence supporting our hypothesis that Akt plays a key role in angiogenic processes. We also observed that exosomes from MSC ${ }^{\mathrm{CR} 4}$ deleting CXCR4 genes by siRNA did not show cardiac protection effects in vitro as well as functional recovery of the infarcted heart in vivo (data not shown), indicating that CXCR4 plays critical role in stem cell functions.

There are several issues to be addressed before exosomebased therapy is widely used in clinical practice. Therefore, further efforts need to (1) identify tissue specific exosome subpopulation; (2) engineer or modify exosome surface antigen and internal content to incorporate large amounts of cardioprotective-proteins or microRNAs (miRNAs); (3) discover the mechanisms of cell uptake; (4) develop efficiency of targeting strategies; (5) develop cost effective methods to generate optimum amount patient-derived exosomes.

The results from in vitro studies showing the proangiogenic effects of $\mathrm{MSC}^{\mathrm{CR} 4}$-derived exosomes were further confirmed by in vivo MI model with cell patch system. Our data indicate that cardiac functions were improved by transplantation of exosome-treated MSC via increased angiogenesis in the infarcted heart. Moreover, exosomes from MSC overexpressing CXCR4 showed better efficiency for reducing left ventricular remodeling and promoting restoration of heart function after MI, confirming that CXCR4 is a key factor for angiogenesis and cell survival. Taken together, this study demonstrated that overexpression of CXCR4 in MSC would be an effective strategy to enhance the release of exosomes containing cardioprotective factors.

\section{Conclusion}

CXCR4-enriched exosomes can be acquired from CXCR4 overexpressing MSC, and treatment with these exosomes can protect cardiomyocytes from ischemic injury both in vitro and in vivo. This novel role of CXCR4-enriched exosomes on cardiac protection highlights a new perspective into intercellular mediation of tissue injury after MI. Upregulation of the Akt signaling pathway contributed to these beneficial effects, suggesting that CXCR4-enriched exosomes may serve as an additional therapeutic strategy to promote cell survival and angiogenesis in ischemic hearts, potentially engendering a novel approach to the development of biologics for repair of MI.

\section{Conflict of Interests}

The authors declare that there is no conflict of interests regarding the publication of this paper.

\section{Authors' Contribution}

Kai Kang and Ruilian Ma contributed equally to this work.

\section{Acknowledgment}

This work was supported by Grants R01HL089824, R01 HL110740, and R01HL-107957 from the National Institutes of Health (Yigang Wang).

\section{References}

[1] C. Nombela-Arrieta, J. Ritz, and L. E. Silberstein, "The elusive nature and function of mesenchymal stem cells," Nature Reviews Molecular Cell Biology, vol. 12, no. 2, pp. 126-131, 2011.

[2] J. Wen, J. Q. Zhang, W. Huang, and Y. Wang, "SDF-1alpha and CXCR4 as therapeutic targets in cardiovascular disease," American Journal of Cardiovascular Disease, vol. 2, no. 1, pp. 20 28, 2012.

[3] D. Zhang, G. C. Fan, X. Zhou et al., "Over-expression of CXCR4 on mesenchymal stem cells augments myoangiogenesis in the infarcted myocardium," Journal of Molecular and Cellular Cardiology, vol. 44, no. 2, pp. 281-292, 2008.

[4] R. C. Lai, T. S. Chen, and S. K. Lim, "Mesenchymal stem cell exosome: a novel stem cell-based therapy for cardiovascular disease," Regenerative Medicine, vol. 6, no. 4, pp. 481-492, 2011.

[5] C. Villarroya-Beltri, C. Gutiérrez-Vázquez, F. Sánchez-Cabo et al., "Sumoylated hnRNPA2B1 controls the sorting of miRNAs into exosomes through binding to specific motifs," Nature Communications, vol. 4, article 2980, 2013.

[6] C. Théry, M. Ostrowski, and E. Segura, "Membrane vesicles as conveyors of immune responses," Nature Reviews Immunology, vol. 9, no. 8, pp. 581-593, 2009.

[7] S. Sahoo, E. Klychko, T. Thorne et al., "Exosomes from human $\mathrm{CD} 34^{+}$stem cells mediate their proangiogenic paracrine activity," Circulation Research, vol. 109, no. 7, pp. 724-728, 2011.

[8] H. W. Kim, H. K. Haider, S. Jiang, and M. Ashraf, "Ischemic preconditioning augments survival of stem cells via miR-210 expression by targeting caspase-8-associated protein 2," The Journal of Biological Chemistry, vol. 284, no. 48, pp. 33161-33168, 2009.

[9] B. Dai, W. Huang, M. Xu et al., "Reduced collagen deposition in infarcted myocardium facilitates induced pluripotent stem cell engraftment and angiomyogenesis for improvement of left ventricular function," Journal of the American College of Cardiology, vol. 58, no. 20, pp. 2118-2127, 2011. 
[10] I. Kadiu, P. Narayanasamy, P. K. Dash, W. Zhang, and H. E. Gendelman, "Biochemical and biologic characterization of exosomes and microvesicles as facilitators of HIV-1 infection in macrophages," Journal of Immunology, vol. 189, no. 2, pp. 744754, 2012.

[11] C. Liu, S. Yu, K. Zinn et al., "Murine mammary carcinoma exosomes promote tumor growth by suppression of NK cell function," Journal of Immunology, vol. 176, no. 3, pp. 1375-1385, 2006.

[12] J. Liang, W. Huang, X. Yu et al., "Suicide gene reveals the myocardial neovascularization role of mesenchymal stem cells overexpressing CXCR4 (MSC(CXCR4))," PLoS ONE, vol. 7, no. 9, Article ID e46158, 2012.

[13] K. Ohashi, T. Yokoyama, M. Yamato et al., "Engineering functional two- and three-dimensional liver systems in vivo using hepatic tissue sheets," Nature Medicine, vol. 13, no. 7, pp. 880-885, 2007.

[14] D. Zhang, W. Huang, B. Dai et al., "Genetically manipulated progenitor cell sheet with diprotin A improves myocardial function and repair of infarcted hearts," The American Journal of Physiology-Heart and Circulatory Physiology, vol. 299, no. 5, pp. H1339-H1347, 2010.

[15] L. Biancone, S. Bruno, M. C. Deregibus, C. Tetta, and G. Camussi, "Therapeutic potential of mesenchymal stem cellderived microvesicles," Nephrology Dialysis Transplantation, vol. 27, no. 8, pp. 3037-3042, 2012.

[16] K. R. Vrijsen, J. P. G. Sluijter, M. W. L. Schuchardt et al., "Cardiomyocyte progenitor cell-derived exosomes stimulate migration of endothelial cells," Journal of Cellular and Molecular Medicine, vol. 14, no. 5, pp. 1064-1070, 2010.

[17] W. Huang, D. Zhang, R. W. Millard et al., "Gene manipulated peritoneal cell patch repairs infarcted myocardium," Journal of Molecular and Cellular Cardiology, vol. 48, no. 4, pp. 702-712, 2010.

[18] R. C. Lai, F. Arslan, M. M. Lee et al., "Exosome secreted by MSC reduces myocardial ischemia/reperfusion injury," Stem Cell Research, vol. 4, no. 3, pp. 214-222, 2010.

[19] R. K. Ganju, S. A. Brubaker, J. Meyer et al., "The $\alpha$-chemokine, stromal cell-derived factor- $1 \alpha$, binds to the transmembrane Gprotein-coupled CXCR- 4 receptor and activates multiple signal transduction pathways," Journal of Biological Chemistry, vol. 273, no. 36, pp. 23169-23175, 1998.

[20] S. Lee, T. T. Chen, C. L. Barber et al., "Autocrine VEGF signaling is required for vascular homeostasis," Cell, vol. 130, no. 4, pp. 691-703, 2007.

[21] T. Yokota, H. Shimokawa, S. Shibata et al., "Insulin-like growth factor I regulates apoptosis in condylar cartilage," Journal of Dental Research, vol. 87, no. 2, pp. 159-163, 2008.

[22] S. J. Riedl and Y. Shi, "Molecular mechanisms of caspase regulation during apoptosis," Nature Reviews Molecular Cell Biology, vol. 5, no. 11, pp. 897-907, 2004. 

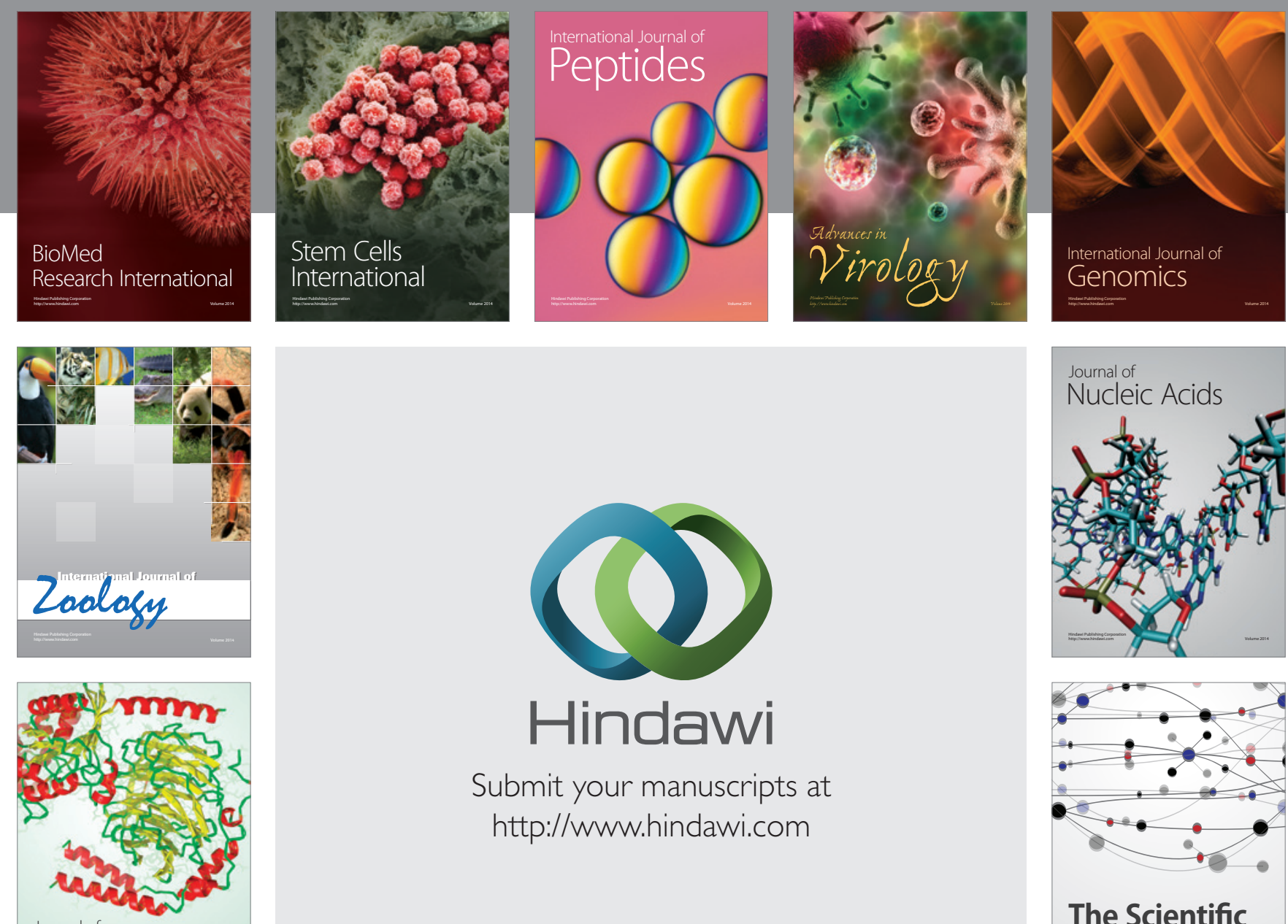

Submit your manuscripts at

http://www.hindawi.com

Journal of
Signal Transduction
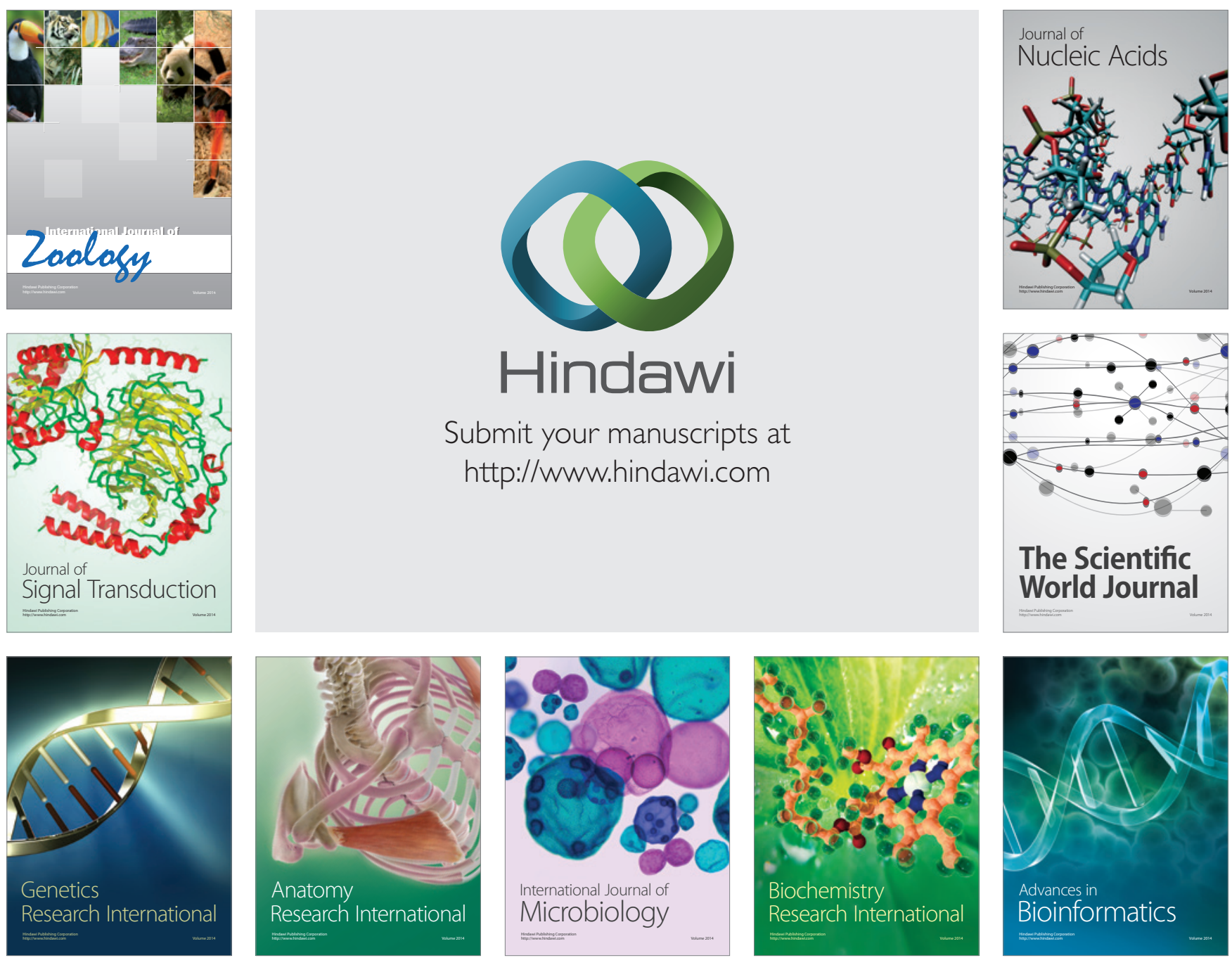

The Scientific World Journal
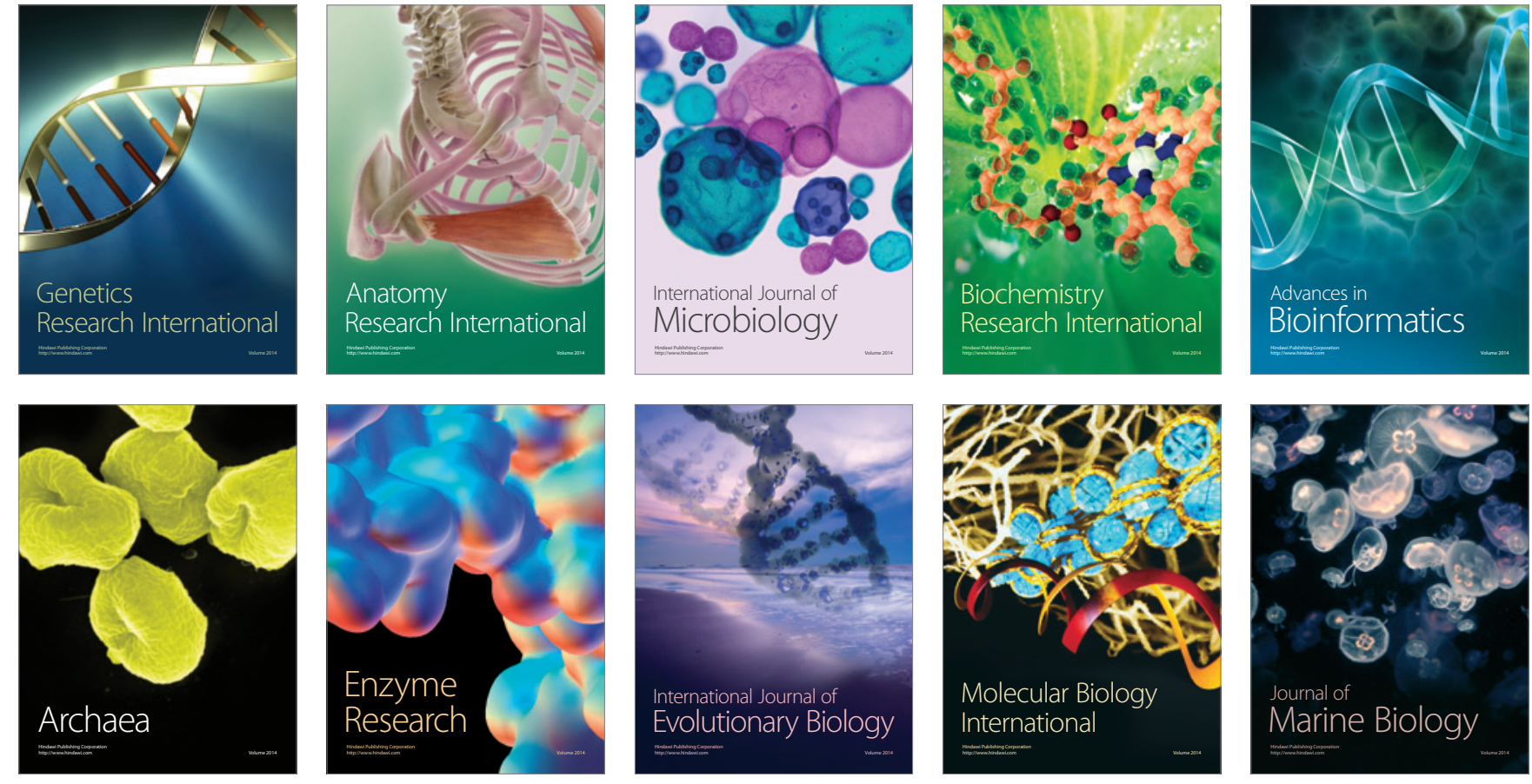